\title{
Comparison of Venous Thromboembolism Prophylactic Measures Post Coronary Artery Bypass Graft Surgery
}

\author{
H. Andrew Wilsey ${ }^{1}$ (D) Komal Pandya ${ }^{1,2} \cdot$ Craig Beavers $^{1,2} \cdot$ Li Xiaoshu $^{3} \cdot$ Ayesha Ather $^{1,2}$
}

Published online: 15 June 2019

○) Springer Nature Switzerland AG 2019

\begin{abstract}
Background There is considerable debate surrounding venous thromboembolism (VTE) prophylaxis in patients post coronary artery bypass grafting (CABG) procedures. The American College of Chest Physicians guidelines report weak recommendations for starting VTE prophylaxis, but provide no specific guidance regarding timing or preferred prophylactic agent. Methods This retrospective cohort study was designed to compare outcomes of post-cardiac surgery patients admitted to the cardiovascular intensive care unit (ICU) who received subcutaneous unfractionated heparin (UFH), with those who received subcutaneous enoxaparin for VTE prophylaxis. Between January 2013 and September 2017, 1085 patients were identified, and, after propensity score matching, 850 patients were selected for analysis. The primary outcomes were postoperative VTE and the occurrence of bleeding events up to 30 days postoperatively. Secondary outcomes included chest tube output, days mechanically ventilated, ICU length of stay, total hospital length of stay, and 30-day readmission rates.

Results During the study period, rates of $2.03 \%$ for VTE events and $1.38 \%$ for bleeding events were reported in the entire cohort. After matching, the rates of VTE events $(2.12 \%$ vs. $1.41 \%, p=0.43)$ and bleeding events $(1.18 \%$ vs. $0.94 \%, p=1.00)$ were more frequent in the heparin group versus the enoxaparin group; these differences were not statistically significant. However, we did find a statistically significant increase in several secondary endpoints, including chest tube output, days mechanically ventilated, ICU length of stay, and total hospital length of stay, within the heparin cohort. Bleeding rates were similar to those previously published, despite the early initiation of VTE prophylaxis.

Conclusions We report no statistical difference in the rates of VTE or bleeding between chemical agents, but our results suggest enoxaparin may be a preferred agent over UFH.
\end{abstract}

\section{Introduction}

The use of chemical venous thromboembolism (VTE) prophylaxis in general surgery is widespread and is supported by strong evidence that suggests a significant reduction in VTE occurrence with the implementation of subcutaneous unfractionated heparin (UFH) or low molecular weight

Electronic supplementary material The online version of this article (https://doi.org/10.1007/s40256-019-00354-4) contains supplementary material, which is available to authorized users.

H. Andrew Wilsey

handreww@buffalo.edu

University of Kentucky HealthCare, Lexington, KY, USA

2 University of Kentucky School of Pharmacy, Lexington, KY, USA

3 Center for Health Service Research, University of Kentucky College of Medicine, Lexington, KY, USA heparin (LMWH) [1]. Patients post coronary artery bypass grafting (CABG) present a significant risk for VTE due to prolonged immobilization, surgical trauma to lower limbs for vein harvest, and activation of inflammatory

\section{Key Points}

In post-coronary artery bypass grafting patients, we found an incidence rate of $2.03 \%$ for venous thromboembolism (VTE) and $1.38 \%$ for bleeding events.

Comparing unfractionated heparin (UFH) with enoxaparin for VTE prophylaxis, we found no statistical difference in the rates of VTE and bleeding.

We found a statistically significant increase in chest tube output, days mechanically ventilated, intensive care unit length of stay, and total hospital length of stay within the UFH cohort. 
and coagulation systems. Cardiac surgery patients also typically carry diagnoses associated with an increased risk of VTE, such as myocardial infarction, diabetes, heart failure, hyperlipidemia, and obesity [2]. Overall, the reported incidence of VTE post CABG surgery varies widely, with estimates ranging from 0.4 to $41 \%$, in part due to symptomdriven versus routine VTE screening methods, diagnostic modalities, and thromboprophylaxis measures [3-5]. The incidence of symptomatic VTE has been described from 0.5 to $3.9 \%$, with deep vein thrombosis (DVT) being more common than pulmonary embolism (PE) [3-6]. Despite these thrombotic risk factors, cardiac surgery patients are also at risk for bleeding complications postoperatively as a result of intraoperative heparin administration and postoperative platelet dysfunction. Based on expert opinion, these patients are at high risk for chemical VTE prophylaxisrelated bleeding, with rates varying from 3.1 to $5.9 \%$ [1]. These factors may further complicate a provider's decision to implement VTE prophylaxis.

Once the decision to implement VTE prophylaxis is made, there is little evidence within the cardiothoracic patient population to suggest a preferred prophylactic agent $[7,8]$. The 2012 American College of Chest Physicians guidelines reflect these limited data, offering a Grade $2 \mathrm{C}$ recommendation for the use of mechanical prophylaxis for uncomplicated CABG, and recommending the additional use of either low-dose UFH or LMWH for prolonged hospitalization [1]. Risk stratification is offered by the 2018 European Society of Anesthesiology (ESA) guidelines for perioperative VTE prophylaxis [9]. These guidelines recommend pharmacological prophylaxis as soon as satisfactory hemostasis has been achieved, in addition to mechanical prophylaxis in patients with one or more risk factors, listed as age above 70 years, transfusion of more than four units of human blood products, mechanical ventilation for more than $24 \mathrm{~h}$, or postoperative complications (acute kidney injury, infection/sepsis, neurological complications). They also provide a Grade $2 \mathrm{C}$ recommendation to use UFH as briefly as possible in favor of LMWHs. Currently, the University of Kentucky Medical Center (UKMC) implements chemical and mechanical VTE prophylaxis immediately postoperatively for patients admitted to the cardiovascular intensive care unit (ICU). The purpose of this study was to describe the frequency of VTE and major bleeding with the use of either subcutaneous UFH or enoxaparin prophylaxis methods post cardiac surgery.

\section{Materials and Methods}

A retrospective cohort study design was used to compare outcomes of patients after cardiac surgery admitted to the cardiovascular ICU who received subcutaneous UFH, with those who received subcutaneous enoxaparin for VTE prophylaxis. This study was approved by the Institutional Review Board at the UKMC. Informed consent was waived as this was a retrospective, observational analysis of adult cardiac surgery patients operated on between 1 January 2013 and 30 September 2017. We included all patients older than 18 years of age who underwent either on-pump or off-pump $\mathrm{CABG}$ in the aforementioned timeframe (see Fig. 1). Exclusion criteria were concurrent valve surgery, early postoperative bleeding within $48 \mathrm{~h}$ of surgery, intravenous heparin within $48 \mathrm{~h}$ from surgery, oral anticoagulation, history of VTE or PE within 3 months of surgery, active malignancy, and Jehovah's Witnesses. Patients were followed for 30 days or until discharge. Upon arrival to the cardiovascular ICU, patients were started on enoxaparin or UFH based on surgeon preference. Heparin was the preferred agent in the presence of renal dysfunction, per enoxaparin manufacturer labeling [10]. To account for treatment crossover, patients were placed in the treatment group based on greatest duration of exposure. The primary outcomes of this study were postoperative VTE and occurrence of bleeding events up to 30 days postoperatively. Postoperative VTE included the cumulative incidence of all VTE events, defined as symptomatic and asymptomatic DVT, and fatal and nonfatal PE. These events were identified by an International Classification of Diseases, Ninth Revision (ICD-9) or ICD, Tenth Revision (ICD-10) diagnosis code of either DVT or PE during the index admission that was confirmed via manual chart review. All computed tomography PE scans and doppler ultrasound orders were also collected and reviewed to capture VTE not previously coded. Evaluation for overt bleeding was investigated in all patients with a $\geq 3 \mathrm{~g} / \mathrm{dL}$ change in daily hemoglobin values, in addition to orders for continuous infusion proton pump inhibitor, recombinant factor VII, prothrombin complex concentrate, or two or more units of packed red blood cells within a 24-h period. Captured bleeding events were then classified by the Bleeding Academic Research Consortium (BARC) criteria. The secondary outcomes included chest tube output, days mechanically ventilated, ICU length of stay, total hospital length of stay, and 30-day readmission rates.

Hypothesis testing was first employed to compare the differences of patient characteristics, treatment outcomes, and presence of comorbidities between the heparin and enoxaparin groups for both the original cohort and matched samples. Patient comorbidities were described using the Elixhauser Comorbidity Index, a method of categorizing comorbidities of patients based on specific ICD diagnosis codes. Categorical variables, summarized using counts and percentages, were analyzed using the Chi-square or Fisher's exact tests. Continuous variables were analyzed using Student's $t$-test, summarized using mean and standard deviation. A propensity score approach was performed to control 


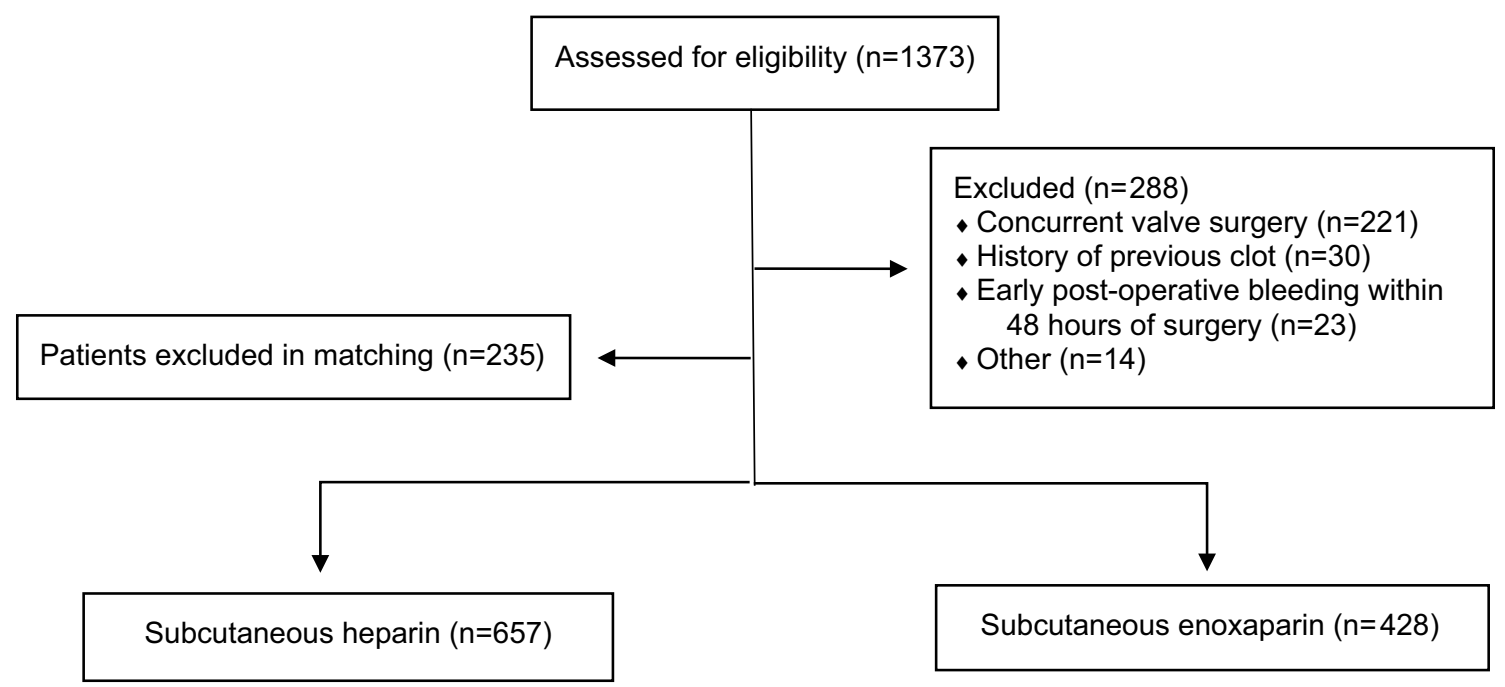

Fig. 1 Patient flow diagram

for observed confounding factors that might influence both group assignment and outcome. Propensity score was defined as the probability of patients treated with enoxaparin based on their individual observed covariates. Probability was estimated based on the logistic regression model, with treatment of enoxaparin as the dependent variable in relation to the following patient characteristics: age, sex, race, height, clopidogrel use, and diagnosis codes of heart failure, hypertension, pulmonary disease, renal failure, coagulopathy and obesity. We employed nearest-neighbor matching and allowed for the exclusion of unpaired data.

\section{Results}

A total of 1085 patients met the inclusion criteria within the set time period, with 657 (60.6\%) patients receiving subcutaneous heparin and 428 (39.4\%) patients receiving subcutaneous enoxaparin. After propensity score matching, we were left with a final cohort of 850 patients between treatment groups. Matched patient baseline characteristics are summarized in Table 1. Of the entire cohort of 1085 patients, a total of 22 VTE events (2.03\%) were identified. Approximately $60 \%$ of patients had two or more events identified, and approximately $75 \%$ of all events occurred within the first 2 weeks from surgery. Only one PE was identified and this patient was included in the heparin cohort. Further details of the thrombotic events are provided in Table 2. A majority of the bleeding events were classified as BARC class IIIa, with only one case of class IIIc bleed described (from the heparin cohort) (Table 3). In our propensity score analysis, the primary outcomes were similar between patients receiving heparin and enoxaparin for both VTE $(2.12 \%$ vs. $1.41 \%, p=0.434)$ and bleeding $(1.18 \%$ vs. $0.94 \%, p=1.00$ ) (Table 4 ). No difference was noted in 30-day readmission rates between cohorts $(6.35 \%$ vs. $5.88 \%$, $p=0.775)$. Patients in the heparin cohort spent significantly more days on the ventilator (1.9 vs. $1.3, p=0.010$ ) and had significantly more daily chest tube output (324 vs. $287 \mathrm{~mL}$, $p<0.001$ ). These patients also had longer ICU length of stay (4.6 days vs. 3.7 days, $p=0.006$ ) and total hospital length of stay (9.0 days vs. 8.0 days, $p=0.009$ ). As matching involves the exclusion of data, we also performed a multivariate analysis on the initial cohort of 1085 patients. After controlling for patient comorbidities and concomitant antiplatelet medications, patients in the enoxaparin cohort still had significantly less time on the ventilator, chest tube output, ICU length of stay, and total hospital length of time (see the Appendix available as electronic supplementary material).

\section{Discussion}

In this single-center, retrospective cohort study of patients post cardiac surgery, we found no statistically significant difference in the rate of VTE or bleeding events between UFH and LMWH prophylaxis. Conversely, prophylaxis with enoxaparin was associated with a significant decrease in chest tube output, days mechanically ventilated, ICU length of stay, and total hospital length of stay.

Currently, the 2012 American College of Chest Physicians guidelines offer a Grade $2 \mathrm{C}$ recommendation for the use of mechanical prophylaxis for uncomplicated CABG over no prophylaxis or chemical prophylaxis [1]. They recommend the addition of pharmacologic prophylaxis to mechanical prophylaxis in cases of prolonged hospitalization, but provide no guidance in defining what constitutes an 'uncomplicated' or 'prolonged' visit, nor do they cite a preferential pharmacologic therapy. The 2018 ESA guidelines 
Table 1 Patient characteristics after propensity score matching

\begin{tabular}{|c|c|c|c|}
\hline Characteristics & Heparin $(n=425)$ & Enoxaparin $(n=425)$ & $P$ value \\
\hline Age, yr & $61.69 \pm 9.92$ & $61.27 \pm 10.61$ & 0.552 \\
\hline Male sex, no. (\%) & $318(74.82)$ & $298(70.12)$ & 0.125 \\
\hline Caucasian race, no. $(\%)$ & $414(97.41)$ & $414(97.41)$ & 1.000 \\
\hline Height, $\mathrm{cm}$ & $172.80 \pm 9.71$ & $172.42 \pm 10.41$ & 0.586 \\
\hline Weight, kg & $87.97 \pm 21.41$ & $89.73 \pm 19.88$ & 0.216 \\
\hline $\mathrm{BMI}^{\mathrm{a}}$ & $29.48 \pm 6.31$ & $30.13 \pm 6.54$ & 0.139 \\
\hline \multicolumn{4}{|l|}{ Comorbidities } \\
\hline \multicolumn{4}{|l|}{ Elixhauser index } \\
\hline Heart failure & $115(27.06)$ & $108(25.41)$ & 0.585 \\
\hline Peripheral vascular disease & $67(15.76)$ & $78(18.35)$ & 0.316 \\
\hline Chronic hypertension & $53(12.47)$ & $49(11.53)$ & 0.673 \\
\hline Chronic pulmonary disease & $201(47.29)$ & $198(46.59)$ & 0.837 \\
\hline Diabetes & $123(28.94)$ & $117(27.53)$ & 0.648 \\
\hline Diabetes with chronic complications & $97(22.82)$ & $106(24.94)$ & 0.469 \\
\hline Renal failure & $34(8.00)$ & $32(7.53)$ & 0.798 \\
\hline Peptic ulcer disease & $3(0.71)$ & $3(0.71)$ & 1.000 \\
\hline Coagulopathy & $152(35.76)$ & $145(34.12)$ & 0.615 \\
\hline Obesity & $139(32.71)$ & $135(31.76)$ & 0.769 \\
\hline \multicolumn{4}{|l|}{ Laboratory parameters } \\
\hline Hematocrit & $28.8 \pm 4.3$ & $29.1 \pm 4.6$ & 0.309 \\
\hline Hemoglobin & $9.6 \pm 1.5$ & $9.7 \pm 1.7$ & 0.412 \\
\hline Platelet & $151.7 \pm 47.7$ & $155.0 \pm 48.9$ & 0.309 \\
\hline Creatinine & $1.1 \pm 0.7$ & $1.0 \pm 0.3$ & $<0.001$ \\
\hline Creatinine clearance ${ }^{\mathrm{b}}$ & $95.5 \pm 38.6$ & $105.0 \pm 44.1$ & $<0.001$ \\
\hline \multicolumn{4}{|l|}{ Medications } \\
\hline Clopidogrel & $86(20.24)$ & $103(24.24)$ & 0.161 \\
\hline Ticagrelor & $4(0.94)$ & $6(1.41)$ & 0.752 \\
\hline
\end{tabular}

Plus-minus valves are means \pm standard deviation

${ }^{\text {a }}$ The body-mass index is the weight in kilograms divided by the square of the height in meters

${ }^{\mathrm{b}}$ Creatine clearance was estimated according to the Cockcroft-Gault formula

provide risk stratification criteria, encourage rapid implementation, and preferentially recommend LMHW over UFH when bleeding risk decreases [9]. The matter is further convoluted by the wide range of VTE rates reported post CABG surgery. Previous reports indicate rates of symptomatic VTE from 0.5 to $3.9 \%$, which have been contrasted by more recent evidence, demonstrating that $13 \%$ of patients receiving VTE prophylaxis still developed silent events, confirmed via computer tomography or ultrasound [3, 4]. A recent survey of national practice patterns reported that $67 \%$ of hospitals currently utilize a combination of pharmacologic and mechanical prophylaxis postoperatively, despite limited guidance [11]. Furthermore, most institutions report initiating chemical VTE prophylaxis on postoperative day 1 or 2 , citing bleeding concerns as the primary reason for delay. A recent systematic review and meta-analysis examined 68 studies providing data on VTE outcomes related to thromboprophylaxis after cardiac surgery [12]. Ho et al. identified a reduced risk of VTE with VTE prophylaxis and no evidence that VTE prophylaxis increases the risk of cardiac tamponade or bleeding. In summary, they recommend to start VTE prophylaxis as soon as possible in those patients without active bleeding. At our institution, chemical VTE orders (either subcutaneous enoxaparin or UFH) are placed on postoperative day zero. All patients have sequential compression devices (SCDs) ordered postoperatively. Furthermore, as a standard of care, all patients are started on aspirin therapy on postoperative day zero, excluding those with a documented allergy.

The effectiveness of VTE prevention therapies was recently evaluated in a large observational cohort of over 90,000 patients who underwent either on-pump or off-pump CABG, with similar exclusion criteria as our current study [13]. The VTE events were captured via discharge diagnosis code for DVT or PE during index admission or subsequent readmission. Kulik et al. found the overall incidence 
Table 2 Venous thromboembolism (VTE) characteristics

\begin{tabular}{lcc}
\hline & $\mathrm{N} / \mathrm{n}(\%)$ \\
\hline Total VTE $(N=22)$ & & \\
Heparin & 16 & 72.7 \\
Deep vein thrombosis & 15 & \\
Pulmonary embolism & 1 & \\
Enoxaparin & 6 & 27.3 \\
Deep vein thrombosis & 6 & \\
Pulmonary embolism & 0 & \\
Number of VTE per patient & & \\
1 & 9 & 40.9 \\
2 & 5 & 22.7 \\
3 & 5 & 22.7 \\
$\geq 4$ & 3 & 13.6 \\
Post-operative day of VTE diagnosis & & \\
$3-7$ & 6 & 27.3 \\
$8-14$ & 11 & 50.0 \\
$15-21$ & 3 & 13.6 \\
$\geq 22$ & 2 & 9.1 \\
\hline
\end{tabular}

Table 3 Events by bleeding academic research consortium (BARC) criteria

\begin{tabular}{lll}
\hline Bleeding events & Heparin $(n=11)$ & $\begin{array}{l}\text { Enoxa- } \\
\text { parin }(n \\
=4)\end{array}$ \\
\hline Class IIIa & 7 & 3 \\
Class IIIb & 3 & 1 \\
Class IIIc & 1 & 0 \\
\hline
\end{tabular}

of symptomatic VTE was $0.74 \%$, with an overall incidence of bleeding events of $1.69 \%$ in patients treated with heparin or LMWH initiated within $48 \mathrm{~h}$ of surgery. Comparatively, we report an overall incidence of VTE of $2.03 \%$ and an overall incidence of bleeding of $1.38 \%$ for patients with VTE initiated upon arrival to the cardiovascular ICU (Table 2). Even with our rapid implementation of VTE prophylaxis, we reported higher VTE rates. Compared with the study by Kulik et al., our baseline population had more frequent comorbidities and a longer length of stay. Our higher VTE rates may be attributed to our high-risk patient population (Table 1), as well as our study methods, which allowed for the capture of asymptomatic events. Importantly, we did not encounter increased hemorrhagic complications, reporting similar rates of bleeding to those previously published, despite the presence of our early implementation VTE prophylaxis protocol. These observations contribute to the scarce body of evidence, and support that VTE prophylaxis utilization immediately post surgery may not increase bleeding rates.

Prophylactic use of heparin and enoxaparin have been compared previously in general surgery patients, however there is limited evidence comparing these agents in the postoperative CABG patient population [13]. A small retrospective study reviewed approximately 200 patients after heart valve surgery, comparing dalteparin with UFH [14]. Bucci et al. report a lower incidence of VTE (4 vs. 11\%) with LMWH versus UFH, as well as bleeding (3 vs. 10\%) and a lower risk of heparin-induced thrombocytopenia (HIT) [3 vs. 6\%]. When comparing heparin versus enoxaparin, we report higher rates of VTE $(2.44 \%$ vs. $1.40 \%, p=0.24)$ and more frequent bleeding events $(1.67 \%$ vs. $0.93 \%, p=0.43)$. Furthermore, we found a statistically significant increase in several secondary endpoints, including chest tube output, days mechanically ventilated, ICU length of stay, and total hospital length of stay (Table 2).

While the global assertion of LMWH superiority for VTE prophylaxis is not supported by current guidelines, several previous studies have demonstrated significantly better efficacy in certain surgical populations, including orthopedic surgery [15]. Planes et al. compared enoxaparin $40 \mathrm{mg}$ once daily with UFH 5000 units every $8 \mathrm{~h}$ in total hip replacement patients and found a significant reduction in VTE rates

Table 4 Clinical outcomes of patients after propensity score matching

\begin{tabular}{llll}
\hline & Heparin $(n=425)$ & Enoxaparin $(n=425)$ & $P$ value \\
\hline $\begin{array}{l}\text { Primary outcomes } \\
\text { Venous thromboembolism }\end{array}$ & $9(2.12)$ & $6(1.41)$ & 0.434 \\
Bleeding & $5(1.18)$ & $4(0.94)$ & 1.000 \\
Secondary outcomes & & & \\
Hospital length of stay & $8.98 \pm 5.99$ & $8.03 \pm 4.48$ & $3.72 \pm 3.65$ \\
ICU length of stay & $4.63 \pm 5.74$ & $1.29 \pm 1.08$ & 0.009 \\
Days mechanically ventilated & $1.85 \pm 4.30$ & $287.41 \pm 138.88$ & 0.010 \\
Chest tube output (mL/day) & $324.34 \pm 146.85$ & $25(5.88)$ & $<0.001$ \\
30-day readmission & $27(6.35)$ & & 0.775 \\
\hline
\end{tabular}

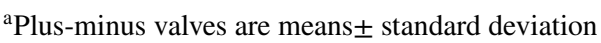


( $25 \%$ to $12.5 \%, p=0.03$ ), as well as fewer red blood cell transfusions and higher hemoglobin values in postoperative days [16]. They concluded that prophylaxis with enoxaparin $40 \mathrm{mg}$ once daily was simple, well-tolerated, and more effective than standard low-dose UFH in their orthopedic patient population.

From a pharmacokinetic and pharmacodynamic view in relation to these chemical agents, there are several considerations that may support our findings. Both agents bind and potentiate their anticoagulant effects via antithrombin activation, mediated by a unique pentasaccharide sequence that induces a conformational change in antithrombin, amplifying its interaction with thrombin and activated factor X (factor Xa) [17]. However, due to their shorter chain length, LMWHs have reduced ability to neutralize thrombin, and thus preferentially act at factor Xa over thrombin. This higher factor Xa to thrombin activity has been suggested to translate to improved efficacy and safety [18]. Additionally, LMWHs exhibit a more predictable anticoagulant response, with less interpatient variability, improved bioavailability, and longer duration of action than UFH [7]. These pharmacokinetic differences have been associated with the reduced propensity of LMWH to bind to plasma proteins, endothelial cells and macrophages [17]. Variations in serum heparin-binding proteins translates to wide ranges of UFH response. This variability may be more significant in post-CABG patients as many of these proteins are acute-phase reactants and are increased in the acute inflammatory process prototypical of invasive cardiac surgery [8]. This predictability of enoxaparin allows for once-daily administration, minimizing disruptions and likely increasing patient satisfaction compared with three injections daily with UFH.

While previous studies evaluating the safety profiles of these agents have found a reduced incidence of bleeding with LMWHs, overall there is limited evidence to claim superior safety $[13,14]$. The reduced bleeding rate associated with LMWHs may also be explained by their reduced affinity for endothelial cells, von Willebrand factor, and platelets. Apart from bleeding, another safety concern is immune-mediated HIT, a prothrombotic adverse drug reaction caused by the development of antibodies to complexes of platelet factor 4 (PF4) and heparin [21]. Of the VTE events reported in this study, none were associated with HIT thrombosis. LMWHs can still cause HIT, however the reduced activation of platelets and release of PF4 with LMWHs translates to a lower incidence of HIT when compared with UFH. Capitalizing on this advantage may be of further value in a patient population that has been described as high risk for the development of HIT [22].

There are several limitations to note. First, the observational retrospective nature of our study is subject to the limitations of ICD-9 and ICD-10 coding that may not have accurately captured all of a patient's disease states, as well as confounding variables that may have influenced our reported outcomes. Due to the risk of drug accumulation, enoxaparin was avoided in patients with renal failure [10]. Thus, those receiving heparin represent an inherently more critically ill population as patients with acute kidney injury, acute or chronic kidney disease, or renal replacement therapy have been independently associated with worse outcomes [23]. However, following sample matching analysis adjusting for baseline demographics, including renal dysfunction, a significant difference in total ICU and hospital length of stay, as well as mechanical ventilator use, were still noted. Second, due to the low incidence of both VTE and bleeding events, our study cohort is not powered to find a difference between heparin and enoxaparin for our primary outcomes. Third, as patients may have been managed at tertiary hospitals for a VTE-related re-admission, we may not have captured the true rates of this secondary outcome. Lastly, our study excluded patients with concurrent valve surgery, as well as early postoperative bleeding within $48 \mathrm{~h}$ of surgery, thus limiting the generalizability of these results.

\section{Conclusions}

Our results from this single-center, retrospective cohort study in patients who are post-CABG found no difference in the rates of VTE or bleeding events between heparin or enoxaparin. Significant differences in several secondary outcomes, including ICU and total hospital length of stay, were noted to be in support of enoxaparin, suggesting enoxaparin may be a preferred agent in this patient population. These results are limited by the retrospective nature of this study, as well as the exclusion criteria, which restricts the generalizability of our findings. We also describe rates of bleeding less than those previously published, suggesting that VTE prophylaxis utilization immediately post-surgery may not increase bleeding rates. These findings contribute to the scarce body of evidence to better assist providers in assessing the risk of early implementation of VTE prophylaxis. Our results have implication for current practice and warrant future research investigating a preferred agent in this postoperative cardiac population.

Acknowledgements The authors would like to acknowledge Kaitlin Musick's role in participating with manual chart review in patients identified to have an event.

\section{Compliance with Ethical Standards}

Funding No external funding was used in the preparation of this manuscript. 
Conflict of Interest H Andrew Wilsey, Komal Pandya, Craig Beavers, Li Xiaoshu, and Ayesha Ather declare they have no potential conflicts of interest that might be relevant to the contents of this manuscript.

\section{References}

1. Gould MK, et al. Prevention of VTE in nonorthopedic surgical patients: antithrombotic therapy and prevention of thrombosis, 9th ed: American College of Chest Physicians Evidence-Based Clinical Practice Guidelines. Chest. 2012;141(2 Suppl):e227S-77S.

2. Protopapas AD, Baig K, Mukherjee D, Athanasiou T. Pulmonary embolism following coronary artery bypass grafting. J Card Surg. 2011;26(2):181-8

3. White RH, Zhou H, Romano PS. Incidence of symptomatic venous thromboembolism after different elective or urgent surgical procedures. Thromb Haemost. 2003;90(3):446-55.

4. Goldhaber SZ, Hirsch DR, MacDougall RC, Polak JF, Creager MA, Cohn LH. Prevention of venous thrombosis after coronary artery bypass surgery (a randomized trial comparing two mechanical prophylaxis strategies). Am J Cardiol. 1995;76(14):993-6.

5. Schwann TA, Kistler L, Engoren MC, Habib RH. Incidence and predictors of postoperative deep vein thrombosis in cardiac surgery in the era of aggressive thromboprophylaxis. Ann Thorac Surg. 2010;90(3):760-8.

6. Hannan EL, et al. Predictors of readmission for complications of coronary artery bypass graft surgery. JAMA. 2003;290(6):773-80.

7. Handeland GF, Abildgaard U, Holm HA, Arnesen KE. Dose adjusted heparin treatment of deep venous thrombosis: a comparison of unfractionated and low molecular weight heparin. Eur J Clin Pharmacol. 1990;39(2):107.

8. Young E, Podor TJ, Venner T, Hirsh J. Induction of the acutephase reaction increases heparin-binding proteins in plasma. Arterioscler Thromb Vasc Biol. 1997;17(8):1568-74.

9. Ahmed AB, Koster A, Lance M, Faraoni D. European guidelines on perioperative venous thromboembolism prophylaxis: cardiovascular and thoracic surgery. Eur J Anaesthesiol. 2018;35(2):84-9.

10. Lovenox (enoxaparin) prescribing information. Bridgewater, NJ: Sanofi-Aventis US LLC; 2013.

11. Mathew SE, Beavers CJ, McNeely E. The national practice patterns of venous thromboembolism prophylaxis post-cardiothoracic surgery. J Pharm Pract. 2017;30(4):394-9.
12. Ho KM, Bham E, Pavey W. Incidence of venous thromboembolism and benefits and risks of thromboprophylaxis after cardiac surgery: a systematic review and meta-analysis. J. Am Heart Assoc. 2015;4(10):e002652.

13. Kulik A, et al. comparative effectiveness of preventative therapy for venous thromboembolism after coronary artery bypass graft surgery. Circ Cardiovasc Interv. 2012;5(4):590-6.

14. Bucci C, Geerts WH, Sinclair A, Fremes SE. Comparison of the effectiveness and safety of low-molecular weight heparin versus unfractionated heparin anticoagulation after heart valve surgery. Am J Cardiol. 2011;107(4):591-4.

15. Koch A, Ziegler S, Breitschwerdt H, Victor N. Low molecular weight heparin and unfractionated heparin in thrombosis prophylaxis: meta-analysis based on original patient data. Thromb Res. 2001;102(4):295-309.

16. Planes A, et al. Prevention of postoperative venous thrombosis: a randomized trial comparing unfractionated heparin with low molecular weight heparin in patients undergoing total hip replacement. Thromb Haemost. 1988;60(3):407-10.

17. Weitz JI. Low-molecular-weight heparins [published erratum in N Engl J Med. 1997;337(21):1567]. N Engl J Med. 1997;337(10):688-98.

18. Ansell J. Factor Xa or thrombin: is factor Xa a better target? J Thromb Haemost. 2007;5(Suppl 1):60-4.

19. Kakkar VV, et al. Efficacy and safety of a low-molecular-weight heparin and standard unfractionated heparin for prophylaxis of postoperative venous thromboembolism: European multicenter trial. World J Surg. 1997;21(1):2-8 (discussion 8-9).

20. Prandoni $\mathrm{P}$, et al. Comparison of subcutaneous low-molecularweight heparin with intravenous standard heparin in proximal deep-vein thrombosis. Lancet. 1992;339(8791):441-5.

21. Warkentin TE, et al. Heparin-induced thrombocytopenia in patients treated with low-molecular-weight heparin or unfractionated heparin. N Engl J Med. 1995;332(20):1330-6.

22. Warkentin TE, Greinacher A. Heparin-induced thrombocytopenia and cardiac surgery. Ann Thorac Surg. 2003;76(6):2121-31.

23. Hashemian SM, et al. Outcome of acute kidney injury in critical care unit, based on AKI network. Tanaffos. 2016;15(2):89-95. 